\title{
Identification and Characterization of Yeast Strains Associated With the Fermented Rice Beverages of Garo Hills, Meghalaya, India
}

\author{
Birendra Kumar Mishra ${ }^{1 *}$, Subrota Hati ${ }^{2}$, Jonali Brahma ${ }^{1}$, Maulik Patel ${ }^{2}$ and Sujit Das ${ }^{1}$ \\ ${ }^{1}$ Department of Rural Development and Agricultural Production, North-Eastern Hill \\ University, Tura campus 794 002, Meghalaya, India \\ ${ }^{2}$ Department of Dairy Microbiology, S.M.C College of Dairy Science, Anand Agricultural \\ University, Anand-388 110, Gujarat, India \\ *Corresponding author
}

\section{A B S T R A C T}

Fermented Rice Beverage is one of the most popular and ethnic fermented alcoholic beverages of Meghalaya Region located in North-eastern part of India. Locally, rice beverage is known as Chubitchi by Garo, kyiad by Khasi and Sadhiar by Jaintias tribes of Meghalaya, it is prepared by using rice and dried starter culture. Dried starter culture or Wanti is used for traditional rice beverage processing by Garo tribes. The nature of microbes was assessed and their source during fermentation was studied generally to explore the microbial diversity associated with the rice beverage samples. Yeast cultures have health attributes and applied in fermented rice beverages since age old. In this study, six yeast cultures were isolated from twenty indigenous fermented food samples collected from various regions of Meghalaya. Isolation was conducted on specific media: MA, SCA, RBA and YPDA for yeast isolates. Based on the phenotypic characteristics obtained from Gram's staining, biochemical characterization of selected isolates was accomplished by API 20 C AUX V5.0 kit for yeast followed by 5.8s rRNA amplification for its genotypic identification and the sequences was deposited at Genebank and NCBI bearing their specific accession numbers were obtained. Further, studies on further techno-functional properties of yeast isolates could be analysed.

\section{Introduction}

Meghalaya is one of the seven states of Northeast India which is bounded to the south by Bangladesh and to the north and east by India's State of Assam. In Meghalaya three major tribes prepare and consume indigenous fermented rice beverage which are known as Chubitchi by Garos, kyiad by Khasi and Sadhiar by Jaintias. Fermented rice beverage is a traditional alcoholic beverage which was formerly exclusive to East Asian and Southeast Asian countries and now is popular in countries like China, Korea, Japan, Philippines, Vietnam and some parts of India. About 220 diversified ethnic tribes of North East India consume rice beer on a regular basis in different forms prepared by their own traditional method by using different starter cultures made of locally available rice and 
medicinal plants (Mishra et al., 2016). It is seen to have important roles in the sociocultural life of the tribal people of North-east India as it is found to be associated with many occasions like ritual ceremonies, marry making, festivals, marriages and even death ceremonies (Saikia et al., 2007). It is now a part of Asian cuisine and is believed to possess many therapeutic and medicinal properties (Teron et al., 2006). They are called as apong in Adi, Laopani in Aka, ijasuijang in Naga, jumai by Bodos, jou by Meches and dimasas, bankchung in Mongpa, Chi in lepcha, morpo by mikris, $z u$ by Tiwas, apong by Mishings, suze by Deoris, laopani and mod by some other tribal communities of Northeast India (Deka and Sarma, 2010). These products are similar to shaosingiji and laochao of China, sake of Japan, brem bali, tape-ketan and tapuy of Indonesia, khaomak of Thailand, chongju and takju of Korea and tapai pulul of Malaysia (Lee CH, 2009), ruou de or ruou nepin Vietnam, Makgeolli in Korea (Kim, et al., 2013, Dung, 2004) etc. In India, an alcoholic beverage called sura, distilled from rice, was in use between 3000 and 2000 B.C. (Eraly, 2002).

Fermented rice beverage is prepared by brewing sticky rice known as Minal by Garo tribe and Ja-Shulia by Khasi tribe by addition of starter culture known as Wanti by Garo tribe and Thiat (natural yeast) by Jaintia Tribe. Starter cultures cakes are generally made from the ground rice powder mixed with medicinal herbs powders which are made into sticky paste and small round cakes are prepared with standard size of $4-5 \mathrm{~cm}$ in diameter and 0.8$1 \mathrm{~cm}$ in thickness. These cakes are exposed to sunlight or tied about 1.20-1.50 m above the fireplace/hearth for drying until the cakes get harden and then are used for rice brewing as natural yeast (Samati et al., 2007). These traditional rice beverages have different compositions according to the formulation and processes used, but the principle of their manufacture can be characterized as a biochemical modification that is saccharification of cereal starches brought about by microorganisms in which fungi (yeasts and moulds) play essential roles. Moulds produce the amylases that degrade the starch into dextrins and sugars, and yeasts convert these sugars to alcohol. The preparation and the use of starter cake as a source of inoculums are important in the manufacture of rice alcoholic beverage. Dried starter cakes normally include yeasts, moulds and bacteria and convert starchy materials to fermentable sugars and subsequently to alcohol and organic acids. The use of different starter cultures with varying microbial content, rice variety and medicinal herbs has been associated with the production of wine with different tastes and flavours, the quantity and quality of wine. Glutinous or sticky rice for instance is a rich source of starch, protein and various microelements that are used by microbes during the fermentation process to produce more wine (Palaniveloo et al., 2013). Among yeasts, Saccharomyces cerevisiae is of industrial importance due to its ability to convert sugars (i.e., glucose, maltose) into ethanol and carbon dioxide (baking, brewing, distillery, and liquid fuel industries). $S$. cerevisiae breaks down glucose through aerobic respiration in presence of oxygen. If oxygen is absent, the yeast will then go through anaerobic fermentation. The net result of this process is two adenosine triphosphate molecules, in addition to two by products; carbon dioxide and ethanol (Mugula et al., 2003).

Different studies on traditionally prepared and isolation of potential yeast from fermented rice beverages have been carried out but molecular level study of isolates from fermented rice beverages from Meghalaya has not yet been reported. Keeping in view the potential health benefits and its nutraceutical properties of probiotics, this study is designed 
to explore the novel yeast, particularly, Saccharomyces spp. from ethnic fermented rice beverages up till molecular level characterization along with phylogenetic studies. In Meghalaya, this study will help to provide valuable functional food with particular health benefits. The aim of this paper was to identify and characterize the predominant species of Saccharomyces spp.in naturally fermented rice beverages of Meghalaya, India. These species were characterized using phenotypic and molecular techniques for confirmation of genus and species level of Saccharomyces spp. strains, along with DNA sequencing and analysis of phylogenetic studies by utilizing 5.8S rRNA gene.

\section{Materials and Methods}

\section{Sample collection and growth enrichment}

Indigenous homemade fermented rice beverages and dried starter rice cakes were collected from different parts of Meghalaya for its analysis in the laboratory (Table 1). Rice beverages were collected in sterile sample container and preserved at $4{ }^{\circ} \mathrm{C}$ for further analysis. Starter rice cakes were grounded to fine powder and kept at $4{ }^{\circ} \mathrm{C}$ for further analysis. The enrichment process was carried out by inoculating approximately 1 $\mathrm{ml} / 1 \mathrm{gm}$ of sample into $50 \mathrm{ml}$ of Yeast peptone dextrose (YPD) broth and incubated at $32^{\circ} \mathrm{C}$ for (2-5) days. All samples were kept in sterile glass bottles at refrigeration temperature (4$6^{\circ} \mathrm{C}$ ) for further analysis.

\section{Identification of yeast strains}

All isolates were serially diluted by adding $1 \mathrm{ml}$ of sample into $9 \mathrm{ml}$ of peptone water up to $10^{-6}$ and streaked on Rose Bengal Agar (RBA), Sabouroud Dextrose Agar (SDA) and Malt's Agar (MA) (Himedia, India). It was incubated at $32^{\circ} \mathrm{C}$ for $2-5$ days. All isolates were tested for catalase activity, Gram's reaction and cell morphology. The identification of strains was performed according to their morphological, cultural and biochemical properties based on their specific characterization (Salazar et. al., 2016). The strains were analysed for the biochemical sugar fermentation using API 20 AUX 5.0 CH kit (HiMedia, India) according to the manufacturer's instructions. Results were scored after incubation at $32^{\circ} \mathrm{C}$ for $24-48-72$ hours. These results were put on the apiweb ${ }^{\mathrm{TM}}$ identification software with database (V5.1) which uses the phenotypic data to predict a species identity. Interpretations of the fermentations profiles were facilitated by comparing all results obtained for the tested isolates with information from the computer aided database, apiweb ${ }^{\mathrm{TM}}$ (https://apiweb.biomerieux.com).

Confirmation of yeast isolates by colony PCR

DNA was isolated using DNA Kit (Himedia, India). The 5.8S-ITS region was amplified by Polymerase Chain Reaction (PCR) using primers (ITS1 and ITS4) (Table 2). Template was prepared by picking freshly grown colony and transferred to Phosphate buffer and was incubated at $32^{\circ} \mathrm{C}$ for 24 hours. PCR was performed of a reaction mixture containing 50 $\mu \mathrm{l}$ of $1.5 \mathrm{mM} \mathrm{MgCl} 2,0.2 \mathrm{mM}$ dNTPs, $1 \mu \mathrm{M}$ of each primer, $0.025 \mathrm{U}$ of $\mathrm{Taq}$ polymerase and $50 \mathrm{ng}$ of yeast DNA. The PCR mixture was initially heated at $94^{\circ} \mathrm{C}$ for 5 minutes followed by cycles of denaturation at $94^{\circ} \mathrm{C}$ for $1 \mathrm{~min}$, annealing $57^{\circ} \mathrm{C}$ for $1 \mathrm{~min}$ and extension was performed at $72^{\circ} \mathrm{C}$ for 4 minutes (Harju. et. al., 2004). The PCR products and their restriction fragments were subjected to electrophoresis for $1 \mathrm{~h}$ at $110 \mathrm{~V}$ in $1 \%$ agarose gels, respectively, which were then stained with ethidium bromide $(14 \mathrm{mg} / \mathrm{ml})$ for visualization of the DNA bands under UV light. Fragment sizes were estimated by 
comparison with DNA size markers (Thermo Fisher Scientific).

\section{Phylogenetic analysis}

In order to determine the closest known relatives of the partial 16S rDNA sequences obtained, in NCBI GenBank, nucleotide database searches were performed and later those particular sequences were processed by multiple sequence alignment tools using the DNA alignment program MAFFT v6.864 for signifying the evolutionary relatedness (Fig. 3) between the yeast strains by UPGMA (Unweighted Pair Group Method with Arithmetic Mean) (Mishra et al., 2017)

\section{Results and Discussion}

The study was conducted to isolate yeasts from ethnic fermented rice beverages from various places of Meghalaya (North eastern region of India) and explore their phenotypic and genotypic characteristics for further, development of value added products by identifying productive microbial strains.

\section{Phenotypic characterization of the isolates}

A total of six yeast strains were isolated from the twenty fermented samples of rice beverages and Wanti obtained from Meghalaya, India (Table 1). The Gram's positive (Fig. 1) and catalase negative isolates from rice beverages and Wanti samples were considered as presumptive yeast cultures (Table 1).

Further, biochemical tests (Fig. 2) of all the isolates were carried out by API 20 AUX 5.0 $\mathrm{CH}$ kit (bioMerieux, India) through sugar fermentation pattern. The result of API test (Table 3 and 4) showed five isolates (NGL3A, NGL4A, NGL1B, RNS4C and RNL1A) as Saccharomyces cerevisiae and RNS1C was identified as Rhodotorula muciliginosa. Out of six isolates, NGL3A showed sugar fermentation of D-Glucose, D-Galactose, DMaltose, D-Saccharose and D-Raffinose. NGL4A and NGL1B also showed positive for Glycerol fermentation along with D-Glucose, D-Galactose, D-Maltose, D-Saccharose and D-Raffinose. RNS4C showed positive for DGlucose, D-Galactose, and Methyl- $\alpha$ DGlucopyranoside, D-Maltose, D-Saccharose and D-Raffinose respectively. RNL1A showed positive for D-Glucose, D-Galactose, Methyl$\alpha$ D-Glucopyranoside, D-Maltose, DSaccharose and D-Raffinose. RNS1C showed sugar fermentation in D-Glucose, D-Xylose, D-Galactose, D-Maltose, D-Saccharose and D-Raffinose.

Hence, from the above stated biochemical results, it was assumed that all the six isolates may be of yeast. Earlier, Sefa-Dedeh et al., (2003) and Chiang et al., (2006) also isolated Saccharomyces cerevisiae, Candida krusei, $C$. pelliculosa, C. glabrata, C. utilis, C. sphaerica, C. magnoliae, Rhodotorula mucilaginosa, $R$. glutinis and Cryptococcus laurentii yeasts from Tapai, a fermented rice beveragein Malaysia. Tamang and Sarkar (1996) reported that Saccharomyces cerevisiae and Lactobacillus spp., was associated with fermentation of Kvass, a rye/wheat-based sour- alcoholic beverage of the Ukraine. Another Indian starter culture used for preparation of for rice wine from Himalayan region (Sikkim and Nepal) called 'Marcha' identified yeast species as Saccharomycopsis capsularis, $S$. bayanus, $P$. anomala, $C$. glabrata, Saccharomycopsis fibuligera, and Pichia burtonii (Tsuyoshi et al., 2005). From this, it can be derived that the yeast associated with rice wine starter culture used in Meghalaya differs from the starter culture used in Sikkim and Nepal. In another study it was also found that non-Saccharomyces yeast like $P$. anomala is connected with frequent cause of spoilage in fermented food and alcoholic beverages (Caggia et al., 2001). 
Table.1 List of selected isolates with their phonotypical characterization

\begin{tabular}{|c|c|c|c|c|c|c|c|c|}
\hline $\begin{array}{l}\text { Sl. } \\
\text { No. }\end{array}$ & $\begin{array}{l}\text { Fermente } \\
\text { d Food } \\
\text { Sample }\end{array}$ & $\begin{array}{l}\text { Tradition } \\
\text { al name of } \\
\text { collected } \\
\text { food } \\
\text { samples }\end{array}$ & $\begin{array}{l}\text { Place of } \\
\text { procuring } \\
\text { fermented } \\
\text { food } \\
\text { samples }\end{array}$ & $\begin{array}{l}\text { Isolate } \\
\text { Code }\end{array}$ & $\begin{array}{l}\text { Morphological } \\
\text { characteristics }\end{array}$ & $\begin{array}{l}\text { Gram' } \\
\text { S } \\
\text { Reactio } \\
\text { n }\end{array}$ & $\begin{array}{l}\text { Catalas } \\
\text { e } \\
\text { Reactio } \\
\text { n }\end{array}$ & $\begin{array}{l}\text { Microscopi } \\
\text { c } \\
\text { Examinati } \\
\text { on }\end{array}$ \\
\hline 1. & $\begin{array}{l}\text { Rice } \\
\text { Beverage }\end{array}$ & Chubitchi & $\begin{array}{l}\text { Chisin a. } \\
\text { Kanang, } \\
\text { north garo } \\
\text { hills, } \\
\text { resubelpara, } \\
\text { Meghalaya }\end{array}$ & $\begin{array}{l}\text { NGL3 } \\
\text { A }\end{array}$ & $\begin{array}{l}\text { Elevated, } \\
\text { circular, entire } \\
\text { and shiny }\end{array}$ & $+v e$ & -ve & $\begin{array}{l}\text { Round to } \\
\text { irregular } \\
\text { shaped in } \\
\text { clusters. }\end{array}$ \\
\hline 2. & $\begin{array}{l}\text { Rice } \\
\text { Beverage }\end{array}$ & Chubitchi & $\begin{array}{l}\text { Chisin a. } \\
\text { Kanang, } \\
\text { north garo } \\
\text { hills, } \\
\text { resubelpara, } \\
\text { Meghalaya }\end{array}$ & $\begin{array}{l}\text { NGL4 } \\
\text { A }\end{array}$ & $\begin{array}{l}\text { Elevated, } \\
\text { circular, entire } \\
\text { and shiny. }\end{array}$ & $+v e$ & -ve & $\begin{array}{l}\text { Round cells } \\
\text { in single, } \\
\text { paired and } \\
\text { some in } \\
\text { clusters }\end{array}$ \\
\hline 3. & $\begin{array}{l}\text { Rice } \\
\text { Beverage }\end{array}$ & Chubitchi & $\begin{array}{l}\text { North garo } \\
\text { hills, } \\
\text { Meghalaya }\end{array}$ & NGL1B & $\begin{array}{l}\text { Small pale pink } \\
\text { circular shiny } \\
\text { entire colony }\end{array}$ & $+v e$ & -ve & $\begin{array}{l}\text { Oval to } \\
\text { circular } \\
\text { cells in } \\
\text { clusters }\end{array}$ \\
\hline 4. & $\begin{array}{l}\text { Starter } \\
\text { Culture }\end{array}$ & Wanti & $\begin{array}{l}\text { Nongkhrah, } \\
\text { nongpoh, } \\
\text { dist ri bhoi, } \\
\text { Meghalaya }\end{array}$ & RNS4C & $\begin{array}{l}\text { Creamy, } \\
\text { glistening, } \\
\text { circular, convex, } \\
\text { entire. }\end{array}$ & $+v e$ & -ve & $\begin{array}{l}\text { Round } \\
\text { small to } \\
\text { medium } \\
\text { cells }\end{array}$ \\
\hline 5. & $\begin{array}{l}\text { Starter } \\
\text { Culture }\end{array}$ & Wanti & $\begin{array}{l}\text { Umtham, } \\
\text { marngar, } \\
\text { nongpoh, } \\
\text { dist ri-bhoi, } \\
\text { Meghalaya }\end{array}$ & RNS1C & $\begin{array}{l}\text { Large circular } \\
\text { dull colony with } \\
\text { rough edges }\end{array}$ & $+v e$ & -ve & $\begin{array}{l}\text { Oval to } \\
\text { elongated } \\
\text { cells with } \\
\text { bud in } \\
\text { single to } \\
\text { clusters. }\end{array}$ \\
\hline 6. & $\begin{array}{l}\text { Rice } \\
\text { Beverage }\end{array}$ & Chubitchi & $\begin{array}{l}\text { Umtham, } \\
\text { marngar, } \\
\text { Nongpoh, } \\
\text { dist ri-bhoi, } \\
\text { Meghalaya }\end{array}$ & RNL1A & $\begin{array}{l}\text { Small circular } \\
\text { smooth edge, } \\
\text { shiny and } \\
\text { elevated colony }\end{array}$ & $+v e$ & -ve & $\begin{array}{l}\text { Round cells } \\
\text { in singles } \\
\text { and clusters }\end{array}$ \\
\hline
\end{tabular}

Table.2 Oligonucleotide sequences of Primers

\section{Primers}

\begin{tabular}{l|l}
\hline ITS1 & 5' , TCCGTAGGTGAACCTGCGG 3' \\
\hline ITS4 & 5' TCCTCCGCTTATTGATATGC 3'
\end{tabular}


Table.3 Biochemical characterization of selected isolates (on the basis of Morphological and Physiological characteristics) through API kit- API 20 C AUX V5.0

\begin{tabular}{|c|c|c|c|c|c|c|c|}
\hline \multirow{2}{*}{\multicolumn{2}{|c|}{ API 20 C AUX }} & 1 & 2 & 3 & 4 & 5 & 6 \\
\hline & & NGL3A & NGL4A & NGL1B & RNS4C & RNS1C & RNL1A \\
\hline 1. & Control & - & - & - & - & - & - \\
\hline 2. & D-Glucose & + & + & + & + & + & + \\
\hline 3. & Glycerol & - & + & + & - & - & - \\
\hline 4. & $\begin{array}{l}\text { Calcium 2-keto- } \\
\text { gluconate }\end{array}$ & - & - & - & - & - & - \\
\hline 5. & L-Arabinose & - & - & - & - & - & - \\
\hline 6. & D-Xylose & - & - & - & - & + & - \\
\hline 7. & Adonitol & - & - & - & - & - & - \\
\hline 8. & Xylitol & - & - & - & - & - & - \\
\hline 9. & D-Galactose & + & + & + & + & + & + \\
\hline 10. & Inositol & - & - & - & - & - & - \\
\hline 11. & D-Sorbitol & - & - & - & - & - & - \\
\hline 12. & $\begin{array}{l}\text { Methyl- } \alpha \text { D- } \\
\text { Glucopyranoside }\end{array}$ & - & + & + & + & - & + \\
\hline 13. & $\begin{array}{l}\text { N-Acetyl- } \\
\text { Glucosamine }\end{array}$ & - & - & - & - & - & - \\
\hline 14. & D-Cellobiose & - & - & - & - & - & - \\
\hline 15. & D-Lactose & - & - & - & - & - & - \\
\hline 16. & D-Maltose & + & + & + & + & + & + \\
\hline 17. & D-Saccharose & + & + & + & + & + & + \\
\hline 18. & D-Trehalose & - & - & - & - & - & - \\
\hline 19. & D-Melezitose & - & - & - & - & - & - \\
\hline 20. & D-Raffinose & + & + & + & + & + & + \\
\hline 21. & $\begin{array}{l}\text { Hyphae/ pseudo } \\
\text { hyphae }\end{array}$ & - & - & - & - & - & - \\
\hline
\end{tabular}

Table.4 Biochemical characterization of selected isolates through API kit (API 20 C AUXV5.0)

\begin{tabular}{|l|l|l|}
\hline SI. No & Strains & Identified organism \\
\hline 1. & NGL3A & Saccharomyces cerevisiae 1 \\
\hline 2. & NGL4A & Saccharomyces cerevisiae 1 \\
\hline 3. & NGL1B & Saccharomyces cerevisiae 1 \\
\hline 4. & RNS4C & Saccharomyces cerevisiae 1 \\
\hline 5. & RNS1C & Rhodotorula mucilaginosa \\
\hline 6. & RNL1A & Saccharomyces cerevisiae 1 \\
\hline
\end{tabular}


Table.5 NCBI GeneBank accession no. of the identified yeast isolates

\begin{tabular}{|l|l|l|l|}
\hline Sl. No & Strains & Partially identified by BLAST & NCBI GeneBank accession no. \\
\hline 1. & NGL3A & Saccharomyces cerevisiae 1 & MG101823 \\
\hline 2. & NGL4A & Saccharomyces cerevisiae 1 & MG101822 \\
\hline 3. & NGL1B & Saccharomyces cerevisiae 1 & MG183703 \\
\hline 4. & RNS4C & Saccharomyces cerevisiae 1 & MG101827 \\
\hline 5. & RNS1C & Rhodotorula mucilaginosa & MG101829 \\
\hline 6. & RNL1A & Wickerhamomyces anomalus & MG183698 \\
\hline
\end{tabular}

Fig.1 Gram staining of the selected isolates

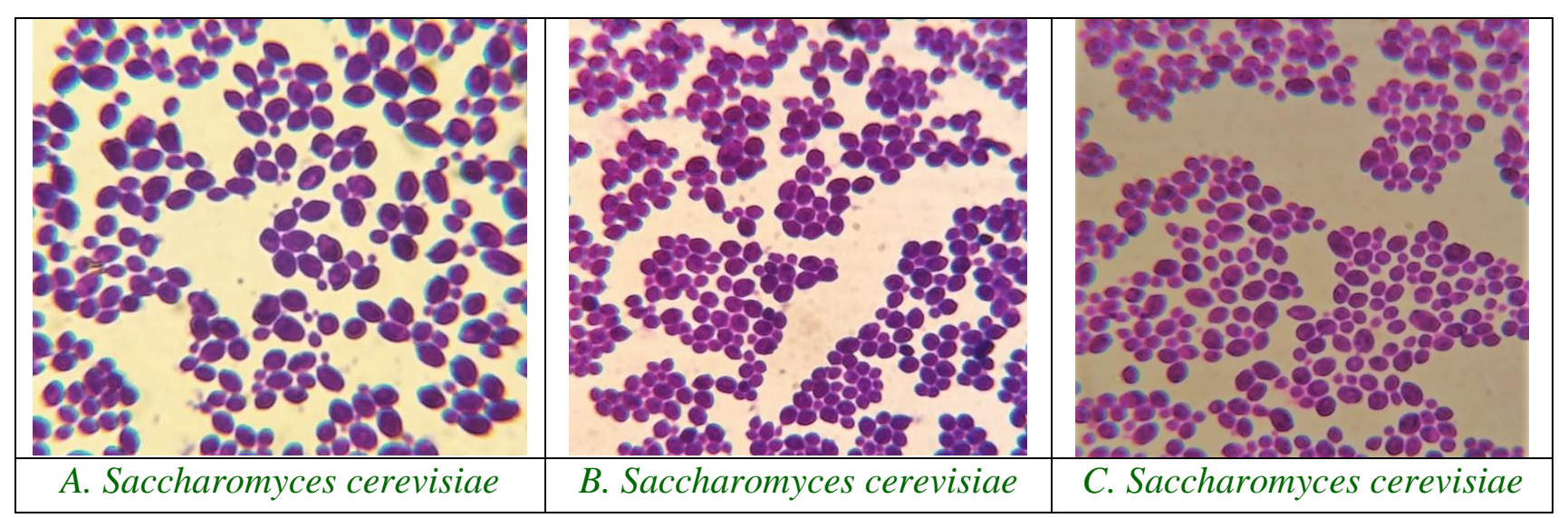

Fig.2 Biochemical analysis of the selected yeast strains

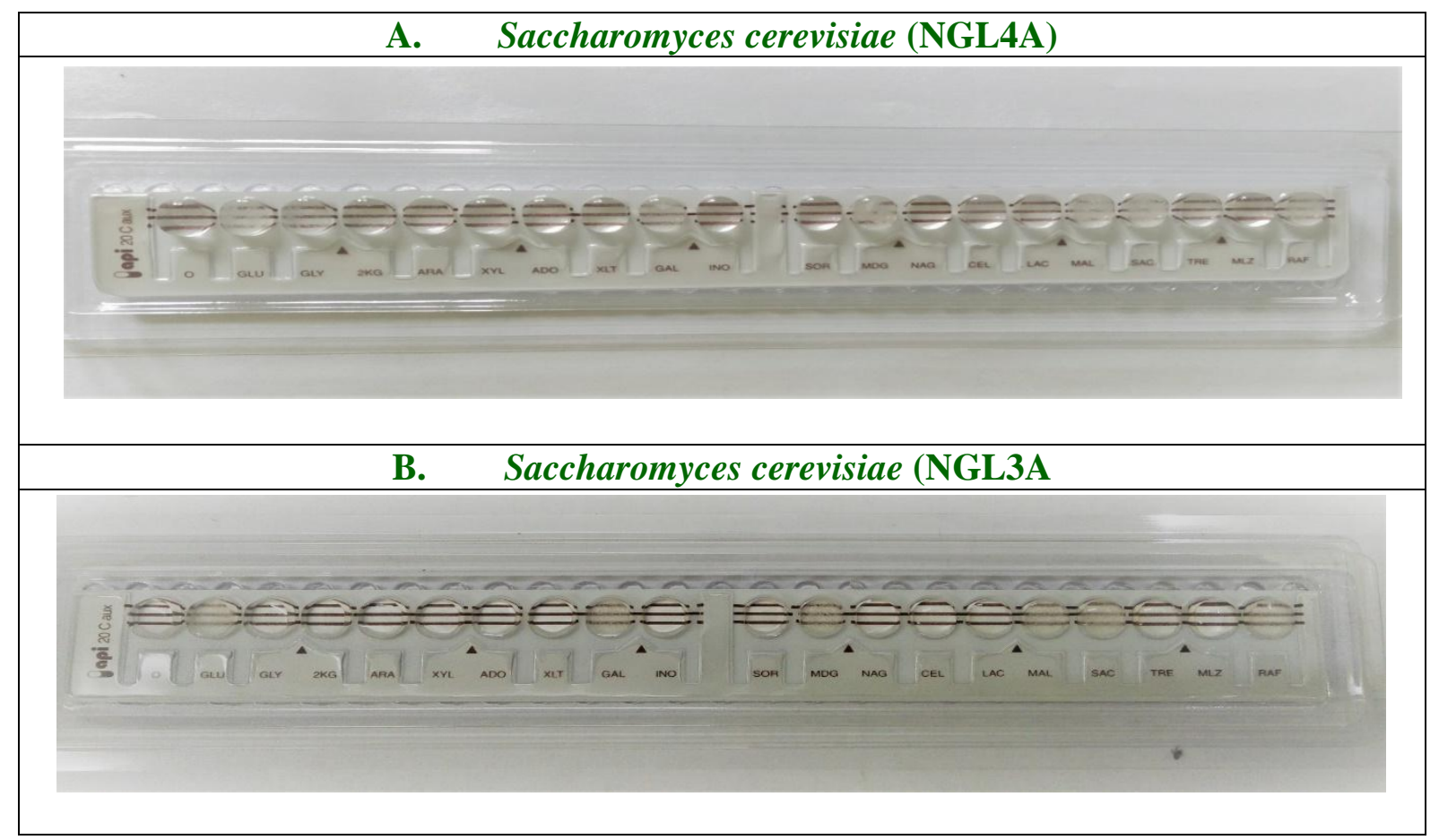


Fig.3 Rooted phylogenetic tree (UPGMA) for the strains of yeast from rice beverages and Wanti

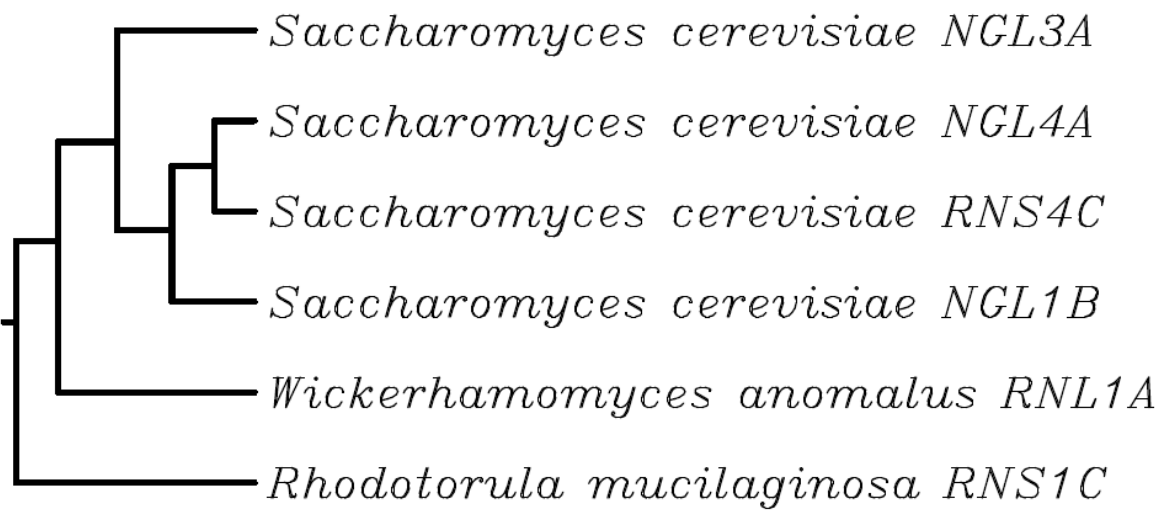

Molecular confirmation and 5.8S rDNA sequence analysis of yeast strains

Strains were identified to the species level by amplification of the 5.8S rDNA gene and flanking Internal Transcribed Spacer (ITS). In the present study, primer ITS1 and ITS4 were used for amplification conserved regions of $5.8 \mathrm{~S}$ rDNA, resulted in product of $>1.5 \mathrm{~kb}$ fragments confirming that the isolate was yeast (Table 2). Similarly, Esteve-Zarzoso et al., (1999) and Lentz et al., (2014) also used 5.8S rDNA for the strains identification upto the species level. The tentative phenotypic identification of all six isolates was confirmed by genotypic characterization in which $5.8 \mathrm{~S}$ rDNA sequence analysis of these isolates as NGL3A, NGL4A, NGL1B and RNS4Cconfirmed the strains $S$. cerevisiae, whereas rest two isolates were identified as $W$. anomalus (RNL1A) and R. Mucilaginosa (RNS4C).The electrophenogram data for $5.8 \mathrm{~S}$ rDNA sequence was validated using Chromas 2.33 software. Sequences obtained were matched with previously published $5.8 \mathrm{~S}$ rDNA sequences of yeast strains available in the GenBank database using BLAST. The sequences determined in this study have been deposited in the NCBI GenBank database with their respective accession numbers (Table 5).

The FASTA sequences of the identified strains after 5.8S rDNA sequence analysis are as follows:

16s rRNA sequence of Saccharomyces cerevisiae (GeneBank Accession no.MG101823)

>MG101823.1, Saccharomyces cerevisiae NGL3A

TTTGGAAATGGATTTTTTTTTGGTTTGG CAAGAGCATGAGAAGCTTTTACTGGGC AAGAAGACAAGAAGATGAGAGGTCAG CCGGGCCTGCGGCTTAAGTGCGCGGTC CTGCTAGGCTTGTAAGTTTCTTTCTTGC TAATGCAAACGGTGAGAGGTTTCTGTG CTTTTGTTATTAGACAAATAAAACCGG TTCAATACAACACACTGTGGAATTTTT CTATCTTTGCCACTTTTTCTTTGGGCAA TCGAGCAATCGGGGCCCCGAGGTTACC AACACCAACAATTTTATTTATTCATTA AATTTTTGTCAAAAAACAAGAATTTTT GTAACCGGAAATTTTTAAATTTTAAAA 
ACTTTCAACAACCGATTTCTTGGTTCTT GCCTCGATGAAGGACGCAGCGAAATT CGATTCGTAAAGTGAATTGCCGAATTC CCTGAATCCTTGAATCTTTGAACGCCC CTTGCGCCCCCTGGTTTTTCCGGGGGC CTGCCTGTTTGAGCGTCCTTTCCTTTTC CAACCTTTTGTTTGGTAGGGAGTGATT CTTTTTGGAGTTAACCTGAAATTGCTG GCCTTTTCCTTGGATGTTTTTTTTTTTC CAAAGAGAGGTTTCTCTGCGTGCTTGA GGTATAATGCAAGTACGGTCGTTTTAG GTTTTACCAACTGCGGCTAATCTTTTTT ATACTGAGCGTATTGGAACGTTATCGA TAAGAAGAGAGCGTCTAGGCGAACAA TGTTCTTAAAGT

16s rRNA sequence of Saccharomyces cerevisiae (GeneBank Accession no. MG101822)

>MG101822.1, Saccharomyces cerevisiae NGL4A

GGAAGGATCATTAAAGAAATTTAATA ATTTTGAAAATGGATTTTTTTTGTTTTG GCAAGAGCATGAGAGCTTTTACTGGGC AAGAAGACAAGAGATGGAGAGTCCAG CCGGGCCTGCGCTTAAGTGCGCGGTCT TGCTAGGCTTGTAAGTTTCTTTCTTGCT ATTCCAAACGGTGAGAGATTTCTGTGC TTTTGTTATAGGACAATTAAAACCGTT TCAATACAACACACTGTGGAGTTTTCA TATCTTTGCAACTTTTTCTTTGGGCATT CGAGCAATCGGGGCCCAGAGGTAACA AACACAAACAATTTTATTTATTCATTA AATTTTTGTCAAAAAACAAGAATTTTC GTAACTGGAAATTTTAAAATATTAAAA ACTTTCAACAACGGATCTCTTGGTTCT CGCATCGATGAAGAACGCAGCGAAAT GCGATACGTAATGTGAATTGCAGAATT CCGTGAATCATCGAATCTTTGAACGCA CATTGCGCCCCTTGGTATTCCGGGGGG CATGCCTGTTTGAGCGTCATTTCCTTCT CAAACATTCTGTTTGGTAGTGAGTGAT ACTCTTTGGAGTTAACTTGAAATTGCT GGCCTTTTCATTGGATGTTTTTTTTTTC
CAAAGAGAGGTTTCTCTGCGTGCTTGA GGTATAATGCAAGTACGGTCGTTTTAG GTTTTACCAACTGCGGCTAATCTTTTTT ATACTGAGCGTATTGGAACGTTATCGA TAAGAAGAGAGCGTCTAGGCGAACAA TGT

16s rRNA sequence of Saccharomyces cerevisiae (GeneBank Accession no. MG183703)

> MG183703.1, Saccharomyces cerevisiae NGL1B

GTAGCGACGCGATCATGATGGTATACT ACTGCTTTCATCAGTACGCTGTGTCCG GAGCAGCGAACGAGTGGATAGTATTA ATTAGTATATGCGTGCAGTATTTCTGT GTGTGCACAATATAGTATAATAAATAT TTGGTGTTTATTCTGCGGGCGCGTGTG TGTGCCACCACAAGAAAAAGTGGCAC AAGTATAAAACCCCCCCTGTGTGTTTT GAAACGTGTTTATATTTCTATAAACAA AACCGCAGAAACTCCCCCTCCTTGTAA CCAGAAGAAAGATTTCTACACTCTAGG AGGCCGGTTTTGGGCGCGCGGGGCGG CGAGACCTCCCCTCTTGTCTCGCGCCA GAAAAAGTCTTCTTCTTGGGAAAAAAA AAAAAAAAAACCCCTTTTACAAAATTA AAAACTTCAAAATTCTTTCTTTCCGGG GGGGGACCTGCGGAGGGATCATTAAA GAAATTTTAATATTTTAGAAAAGGGTT TTTTTTTTTGTTTCGGCAGGAGCATGA GAGCTTTTACGGGGCAAGACGACAAG AGATGGAGACTCCACCCGCGCCTGCGC TTAAGTCGGCGCTCTTGCTAGGCTAAT AATTTTCTTTCTTGTTATTCCAAGCGGG GAGAGATTTGTGTGCTTTTGTTATAGG ACAAATAAACCCGTTTCAATACCACCC CCGGGGGAGTTTTCTTTTTTTTGCAACT TTTTTTTGGGGCATTGGAACAATGGGG CCCCGGAGGTAACACACACAAACAAT TTTTTTTCTTCATAAATTTTTTGTAAAA AACAAGAATTTTTGTAACTGGAAATTT TAAAATATTAAAAACTTTCAACAACGG ATCTCTTGGTTCTCGCATCGAAGAAGA 
ACGCAGCGAAATGCGATACGTAATGT GAATTGCAGAATTCCGTGAATCATCGA ATCTTTGAACGCACATTGCGCCCCGTG GTATTCCGGGGGGCACGCCTGTTTGAG CGTCATTTCCTTCTCAAACATTCTGTTT GGTAGTGAGTGATTCTCTGGGGAGTTA ACTAGAAATTGCTGGCCTTTTCATGGG ATGTTTTTTTTTCCAAAGAGAGGTTTCT CTGCGTGCTTGAGGTATAATGCAAGTA CGGTCGTTTTAGGTTTTACCAACTGCG GCTAATCTTTTTTATACTGAGCGTATTG GAACGTTATCGATAAGAAGAGAGCGT CTAGGCGAACAATGTTCTAAAGTTGAC CTCAATCAGTACGATATCCGTTCCCCT

16s rRNA sequence of Saccharomyces cerevisiae (GeneBank Accession no.MG101827)

> MG101827.1, Saccharomyces cerevisiae RNS4C

GGATTTTTTTTGTTTTGGCAAGAGCAT GAGAGCTTTTACTGGGCAAGAAGACA AGAGATGGAGAGTCCAGCCGGGCCTG CGCTTAAGTGCGCGGTCTTGCTAGGCT TGTAAGTTTCTTTCTTGCTATTCCAAAC GGTGAGAGATTTCTGTGCTTTTGTTAT AGGACAATTAAAACCGTTTCAATACAA CACACTGTGGAGTTTTCATATCTTTGC AACTTTTTCTTTGGGCATTCGAGCAAT CGGGGCCCAGAGGTAACAAACACAAA CAATTTTATTTATTCATTAAATTTTTGT CAAAAAACAAGAATTTTCGTAACTGG AAATTTTAAAATATTAAAAACTTTCAA CAACGGATCTCTTGGTTCTCGCATCGA TGAAGAACGCAGCGAAATGCGATACG TAATGTGAATTGCAGAATTCCGTGAAT CATCGAATCTTTGAACGCACATTGCGC CCCTTGGTATTCCGGGGGGCATGCCTG TTTGAGCGTCATTTCCTTCTCAAACATT CTGTTTGGTAGTGAGTGATACTCTTTG GAGTTAACTTGAAATTGCTGGCCTTTT CATTGGATGTTTTTTTTTTCCAAAGAG AGGTTTCTCTGCGTGCTTGAGGTATAA TGCAAGTACGGTCGTTTTAGGTTTTAC
CAACTGCGGCTAATCTTTTTTATACTG AGCGTATTGGAACGTTATCGATAAGAA GAGAGCGTCTAG

16s rRNA sequence of Rhodotorula mucilaginosa (GeneBank Accession no.MG101829)

> MG101829.1, Rhodotorula mucilaginosa RNS1C

CTAATGATCCTTCCGTAGGTGAACCTG CGGAAGGATCATTAGTGAATATAGGA CGTCCAACTTAACTTGGAGTCCGAACT CTCACTTTCTAACCCTGTGCACTTGTTT GGGATAGTAACTCTCGCAAGAGAGCG AACTCCTATTCACTTATAAACACAAAG TCTATGAATGTATTAAATTTTATAACA AAATAAAACTTTCAACAACGGATCTCT TGGCTCTCGCATCGATGAAGAACGCAG CGAAATGCGATAAGTAATGTGAATTGC AGAATTCAGTGAATCATCGAATCTTTG AACGCACCTTGCGCTCCATGGTATTCC GTGGAGCATGCCTGTTTGAGTGTCATG AATACTTCAACCCTCCTCTTTCTTAATG ATTGAAGAGGTGTTTGGTTTCTGAGCG CTGCTGGCCTTTACGGTCTAGCTCGTT CGTAATGCATTAGCATCCGCAATCGAA CTTCGGATTGACTTGGCGTAATAGACT ATTCGCTGAGGAATTCTAGTCTTCGGA CTAGAGCCGGGTTGGGTTAAAGGAAG CTTCTAATCAGAATGTCTACAT

16s rRNA sequence of Wickerhamomyces anomalus (GeneBank Accession no.MG183698)

> MG183698.1, Wickerhamomyces anomalus RNL1A

GGCAATAGAATACTATAATGATCCTTC CGTAGGTGAACCTGCGGAAGGATCATT ATAGTATTCTATTGCCAGCGCTTAATT GCGCGGCGATAAACCTTACACACATTG TCTAGTTTTTTTGAACTTTGCTTTGGGT AGGCTTTTATGGCTTGCCCAGAGGACA 
ACTAAACACATTTTTTACAAATGTTTT AAACCTTTAACCAATAGTCATGAAAAT TTTTAACAAAATTAAAATCTTCAAAAC TTTCAACAACGGATCTCTTGGTTCTCG CAACGATGAAGAACGCAGCGAAATGC GATACGTATTGTGAATTGCAGATTTTC GTGAATCATCGAATCTTTGAACGCACA TTGCACCCTCTGGTATTCCAGAGGGTA TGCCTGTTTGAGCGTCATTTCTCTCTCA AACCTTTGGGTTTGGTATTGAGTGATA CTCTGTTAAATAGGGTTAACTTGAAAT AATGTCTTAGCAAGAGTGTACTAATTT ATACGTCTTTCTGAAATAATGTATTAG GTTCTTCCAACTCGTTATATCAGCTAG GCAGATGAATAGTATTTTAGGCTCGGC TTAACAATTAAACTAAAAGT

\section{Phylogenetic analysis}

To determine the closest known relatives of the partial 5.8S rDNA sequences obtained, nucleotide database searches were performed in NCBI GenBank and later the sequences were analysed by multiple sequence alignment tools using the DNA alignment program MAFFT v6.864 to signify the evolutionary relatedness between the strains by UPGMA (Unweighted Pair Group Method with Arithmetic Mean). From the phylogram as depicted in Figure 3, it can be depicted that the two isolates, S. cerevisiae (NGL4A and RNS4C) are closely related due to the sequence similarity match as well the nodal distance which in turn is significantly related to $S$. cerevisiae (NGL1B) distantly connected to $S$. cerevisiae (NGL3A). This branch is again distantly related to $W$. anomalus (RNL1A) followed by $R$. mucilaginosa (RNS4C) as represented by the branch length. Each node with descendants represents the inferred most recent common ancestor of the descendants which in this case is Saccharomyces spp.

The present study concluded that $S$. cerevisiae was predominant yeast in microflora of rice beverages and starter culture. The tentative phenotypic identification of these six isolates were confirmed by the phenotypic as well as genotypic (5.8S rDNA sequence analysis) identification which derived that four isolates viz. NGL3A, NGL4A, NGL1B and RNS4C belonged to Saccharomyces cerevisiae, RNL1A as Wickerhamomyces anomalus and RNS4C as Rhodotorula mucilaginosa. Later, phylogenetic tree of the most closely related yeast isolates have been constructed by using MAFFT sequence alignment tool. Further, the isolated strains could be checked for their specific probiotic attributes and can be exploited for the development of value added fermented foods which will be appropriate for glutinous rice fermentation and can improve the quality of traditional rice wine production.

\section{Acknowledgements}

The authors are grateful to the Department of Biotechnology (DBT), Ministry of Science and Technology, New Delhi, India for the financial assistance under Twining Project 2016 in collaboration with Anand Agricultural University, Anand, Gujarat, India and North-Eastern Hill University, Tura Campus, Meghalaya, India.

\section{References}

Caggia, C., Restuccia, C., Pulvirenti, A., Giudici, P. 2001. Identification of Pichia anomala isolated from yoghurt by RFLP of the ITS region. International Journal of Food Microbiology. 71: 71-73.

Chiang, Y. W., Chye, F. Y., and Mohd Ismail, A. 2006. Microbial diversity and proximate composition of Tapai, A Sabah's fermented beverage. Malaysian Journal of Microbiology. 2(1): 1-6.

Deka, D. and Sarma, G.C. 2010. Traditionally used herbs in the preparation of rice-based by the Rabha tribes of Goalpara district, Assam. Indian Journal of Traditional Knowledge. 9(3): 459-462. 
Eraly, A. 2002. Gem in the Lotus. Penguin books limited. pp 165.

Esteve-Zarzoso, B., Belloch, C., Uruburu, F. and Querol, A. 1999. Identification of yeasts by RFLP analysis of the 5.8S rRNA gene andthe two ribosomal internal transcribed spacers. International Journal of Systematic Bacteriology. 49:329-337.

Harju S., Fedosyuk H. and Peterson K.R. 2004. Rapid isolation of yeast genomic DNA: Bust n' Grab. BMC Biotechnology.4:8.

Lee, C.H. 2009. Food Science and Technology. In: Food Biotechnology (Ed.) Campbell Platt, G. John Wiley and Sons, West Sussex, United Kingdom. Pp. 85-95.

Lentz, M., Putzke, T., Hessler, R., and Luman, E. 2014. Genetic and physiological characterization of yeast isolated from ripe fruit and analysis of fermentation and brewing potential. Journal of the Institute of Brewing. 120(4): 559-564.

Mishra, B.K., Balamurugan, N., Hati, S. and Paul, B., 2015. 'Chubitchi'- The native fermented rice beverage of the garos' of west Garo hills, Meghalaya. International Journal of Fermented Foods. 4(1-2): 81-90.

Mishra, B.K., Hati, S., Das, S., and Patel, K. 2017.Bio-Diversity of Lactobacillus Cultures Associated with the Traditional Ethnic Fermented Foods of West Garo Hills, Meghalaya, India. International Journal of Current. Microbiology andApplied Sciences. 6(2): 1090-1102

Mugula, J.K., Nnko, S.A.M., Narvhus, J.A. and Sorhaug, T., 2003. Microbiological and Fermentation characteristics of togwa, a Tanzanian fermented food. International Journal of Food Microbiology. 80: 187-199.
Palaniveloo, K. and Vairappan, C.S. 2013. Biochemical properties of rice wine produced from three different starter cultures. Journal of Tropical Biology and Conservation. 10: 31-41.

Saikia, B., Tag, H. and Das, A.K. 2007. Ethnobotany of foods and beverages among the rural farmers of Tai Ahom of North Lakhimpur district, Asom. Indian Journal of Traditional Knowledge. 6(1): 126-132.

Salazar, M., Humberto Salem, A.Z.M., Kholif Ahmed Monroy, H., Pérez, L.S., Zamora, J.L., Gutiérez, A. 2016. Yeast: description and structure. 4-13.

Samati, H., and Begum, S.S.2007. Kiad-A popular local liquor of Pnartribe of Jaintia hills district, Meghalaya. Indian Journal of Traditional Knowledge. 6(1):133-135.

Sefa-Dedeh, S., Cornelius, B. Amoa-Awua, W. Sakyi-Dawson, E. and Afoakwa, E.O. 2004. The microflora of fermented nixtamalized corn. International Journal of Food Microbiology.96:97-102.

Tamang, J.P. and Sarkar, P.K. 1996. Microbiology of mesu, a traditional fermented bamboo shoot product. International Journal of Food Microbiology. 29: 49-58.

Tsuyoshi, N., Fudou, R., Yamanaka, S., Kozaki, M., Tamang, N., Thapa, S., and Tamang, J.P. 2005. Identification of yeast strains isolated from marcha in Sikkim, a microbial starter for amylolytic fermentation. International Journal of Food Microbiology. 99: 135-146.

\section{How to cite this article:}

Birendra Kumar Mishra, Subrota Hati, Jonali Brahma, Maulik Patel and Sujit Das. 2018. Identification and Characterization of Yeast Strains Associated With the Fermented Rice Beverages of Garo Hills, Meghalaya, India. Int.J.Curr.Microbiol.App.Sci. 7(02): 3079-3090. doi: https://doi.org/10.20546/ijcmas.2018.702.371 